Cite as: Lomas, T., Medina, J. C., Ivtzan, I., Rupprecht, S., \& Eiroa-Orosa, F. J. (2017). The impact of mindfulness on the wellbeing and performance of educators: A systematic review of the empirical literature. Teaching and Teacher Education, 61, 132-141. http://doi.org/10.1016/j.tate.2016.10.008

\title{
The impact of mindfulness on the wellbeing and performance of educators: A systematic review of the empirical literature.
}

\section{Authors}

Tim Lomas $^{1 \dagger}$, Juan Carlos Medina ${ }^{2}$, Itai Ivtzan ${ }^{1}$, Silke Rupprecht $^{3}$, Francisco Eiroa-Orosa ${ }^{1}$

${ }^{1}$ School of Psychology, University of East London, Arthur Edwards Building, Water Lane,

London, E15 4LZ, United Kingdom

${ }^{2}$ Faculty of Psychology, University of Barcelona, Passeig de la Vall d'Hebron, 08035 Barcelona, Spain

${ }^{3}$ Leuphana University, Scharnhorststraße 1, 21335 Lüneburg, Germany

${ }^{\dagger}$ Author responsible for correspondence:

Email: t.lomas@uel.ac.uk

Note: This article may not exactly replicate the final version published in Teaching and Teacher

Education. It is not the copy of recor 


\begin{abstract}
Given the potentially demanding nature of teaching, efforts are underway to develop practices that can improve the wellbeing of educators, including interventions based on mindfulness meditation. We performed systematic review of empirical studies featuring analyses of mindfulness in teaching contexts. Databases were reviewed from the start of records to January 2016. Eligibility criteria included empirical analyses of mindfulness and wellbeing outcomes acquired in relation to practice.

A total of 19 papers met the eligibility criteria and were included in the systematic review, consisting of a total 1,981 participants. Studies were principally examined for outcomes such as burnout, anxiety, depression and stress, as well as more positive wellbeing measures (e.g., life satisfaction).

The systematic review revealed that mindfulness was generally associated with positive outcomes in relation to most measures. However, the quality of the studies was inconsistent, and so further research is needed, particularly involving high-quality randomised control trials.
\end{abstract} Keywords: mindfulness; meditation; education professionals; wellbeing; systematic review 


\section{Introduction}

There are widespread concerns about the increasingly stressful nature of many professions. This claim is based upon the observation that although the prevalence of mental illness in the general United Kingdom (UK) population has not significantly increased in the last twenty years (Office forNational Statistics [ONS], 2014), since 2009 the number of sick days lost to stress, depression and anxiety has increased by $24 \%$, while the number lost to serious mental illness has doubled (Davies, 2014). As the annual report by Sally Davies (2014), the UK's Chief Medical Officer elucidates, mental ill health is the leading cause of sickness absence in the UK, accounting for 70 million sick days (more than half of the 130 million total every year); indeed, each year between 2010 and 2014, a million workers in the UK took sick leave for longer than four weeks. Stress and mental disorders connected to work are a serious problem - obviously for the sufferers themselves, but also for their employers and the wider economy. Davies reports that the indirect costs to the UK of mental ill health in unemployment, absenteeism and presenteeism (leading to loss of productivity) are estimated at between $£ 70$ and $£ 100$ billion, with $£ 9$ billion being paid by employers in terms of sick pay and related costs.

Some jobs are often regarded as particularly stressful. Teaching is widely-regarded as one such profession. Even in countries where it is a well-respected and remunerated occupation, such as Finland (Tirri, 2011), it can still often be a demanding and challenging endeavour, physically, emotionally, cognitively and socially (Blomberg \& Knight, 2015). Moreover, these "inherent" challenges are frequently exacerbated by "external" factors, such as politically-driven structural changes and pressures. In the UK, for instance, a recent survey of 3,500 members of the NASUWT (National Association of Schoolmasters Union of Women Teachers) union - a large UK union for teachers and head teachers, comprising over 300,000 members - found that over two-thirds of respondents had considered leaving 
the profession in the last 12 months (Precey, 2015). The findings revealed the extent to which respondents felt their wellbeing had been adversely affected by work: $83 \%$ reported experiencing workplace stress, while $67 \%$ stated that their job had adversely affected their mental or physical health (with 5\% actually being hospitalised as a result). Arguably, much of this pressures relates specifically to the current context of teaching in the UK (e.g., systemic pressures in the UK education system). The top concerns cited by respondents as being responsible for their work-related stress was workload (flagged up by $89 \%$ of respondents), followed by pay (45\%), inspections (44\%), and curriculum reform (42\%).

Given the burdens of work-related stress - both in teaching, and more generally there is an increasing recognition of the need to take preventative action to mitigate or ameliorate work-related mental health issues (George, Dellasega, Whitehead, \& Bordon, 2013). Some efforts are structural, such as initiatives to provide more flexible working arrangements (Joyce, Pabayo, Critchley, \& Bambra, 2010). Other remedial actions focus more on offering clinical and psychotherapeutic help to staff who may be in need; however, workers may be somewhat reluctant to avail themselves of such services, wary lest it appear on their record or prove detrimental career-wise in some way (Chew-Graham, Rogers, \& Yassin, 2003). Arguably less problematic are interventions and programmes aimed at alleviating or protecting against issues such as stress. (There may be less of a stigma about attending these kind of programmes, since they are often targeted at staff more "generally," rather than specific individuals.) Such initiatives can still prove difficult to implement of course; e.g., staff may be reluctant to engage in these due to perceived lack of time (Bearse, McMinn, Seegobin, \& Free, 2013). However, they are nevertheless increasingly common. In recent years, among the most prominent of these types of initiatives are programmes based around mindfulness meditation - mindfulness-based interventions (MBIs) - which is the focus of this review. 
Before introducing mindfulness, it is worth noting that many such interventions are not only aimed at ameliorating mental health issues, such as anxiety, but promoting wellbeing in a broader sense. Of course, wellbeing is a contested term, used in different ways in various contexts (deChavez, Backett-Milburn, Parry, \& Platt, 2005). For instance, Cooke, Melchert, and Connor (2016) identified four prominent conceptualisations of wellbeing: (1) hedonic wellbeing, also known as 'subjective wellbeing' (Diener, 2000), which encompasses constructs like positive affect and life satisfaction;(2) eudaimonic wellbeing, also known as 'psychological wellbeing' (Ryff, 1989), which includes considerations such as meaning in life; (3), quality of life (Frisch, Cornell, Villanueva, \& Retzlaff, 1992), which often encompasses both hedonic and eudaimonic processes; and (4) 'wellness,' which tends to be used interchangeably with quality of life.

In addition, other conceptualisations of wellbeing emphasise its multidimensional nature. For instance, Pollard and Davidson (2001, p.10) define wellbeing as 'a state of successful performance across the life course integrating physical, cognitive and socialemotional function.' (In constructing wellbeing as being multidimensional in this way, such definitions align with influential multidimensional conceptualisations of health, such as Engel's (1977) biopsychosocial model, and the World Health Organization's (1948) inclusive definition of health as 'a state of complete physical, mental and social well-being, and not merely the absence of disease and infirmity'.) As such, in the current review, we are not only interested in the amelioration of mental health issues, but also in the promotion of 'positive' wellbeing. Thus, our analysis will consider outcomes pertaining to all four conceptualisations identified by Cooke et al. (2016), including hedonic constructs (e.g., positive affect) and eudaimonic constructs (e.g., meaning in life). We shall also look to appraise wellbeing in a multidimensional way, e.g., encompassing health and relationships. With that in mind, let's consider what mindfulness is. 


\section{Mindfulness}

The past few decades have seen a burgeoning interest in mindfulness in the West, spanning clinical practice, academia, and society more broadly. Mindfulness is generally regarded as having originated in the context of Buddhism around the $5^{\text {th }}$ millennium B.C., though its roots stretch back at least as far as the third millennium B.C. as part of the Brahmanic traditions in India, from which Buddhism subsequently emerged (Cousins, 1996). However, it came to prominence in the West particularly through the work of KabatZinn (1982), who harnessed it for an innovative "mindfulness-based stress reduction" (MBSR) programme (discussed further below) which was successfully used to treat chronic pain. Somewhat confusingly, the term "mindfulness" is frequently used to refer to both: (1) a state or quality of mind; and (2) a form of meditation that enables one to cultivate this particular state/quality. Both uses will be deployed in this review, though the context will make clear which particular usage is being used.

In terms of (1), the most prominent and influential operationalisation of mindfulness as a state/quality of mind is Kabat-Zinn's (2003, p.145) widely-cited definition, which constructs mindfulness as "the awareness that arises through paying attention on purpose, in the present moment, and nonjudgmentally to the unfolding of experience moment by moment." Expanding on this idea, Shapiro, Carlson, Astin, and Freedman (2006) formulated a theoretical elucidation of Kabat-Zinn's (2003) definition, deconstructing it into three key "axioms" or components: intention (i.e., a teleological motivation for paying attention in this

way, e.g., a commitment to psychological development); attention (i.e., the cognitive processes and mechanisms through which said attention is enacted); and attitude (i.e., the emotional qualities with which one imbues one's attentive focus, like compassion).

The second main usage of the term mindfulness is for the forms of meditation practice which may facilitate this "mindful" state/quality of mind. Mindfulness meditation, and 
meditation more broadly, refer to a diverse spectrum of mental activities, which share a common focus on training the self-regulation of attention and awareness (Author et al., 2015a), with the goal of enhancing voluntary control of mental processes, thereby increasing wellbeing (Walsh \& Shapiro, 2006). Lutz, Slagter, Dunne, and Davidson (2008) offer a useful way of differentiating between types of meditation, suggesting that most common forms can be identified as featuring either "focused attention" or "open- monitoring" processes. Focused attention can be operationalised in terms of the co-ordination of various attention networks (Mirsky, Anthony, Duncan, Ahearn, \& Kellam, 1991; Posner \& Petersen, 1990), including sustained attention (e.g. towards a selected target, like the breath), executive attention (e.g., preventing one's focus from "wandering"), attention switching (e.g., disengaging from distractions), selective attention and attention re-orienting (e.g. redirecting focus back to the target), and working memory (Lutz et al., 2008; Vago \& Silbersweig, 2012). In contrast, open-monitoring refers to a broader receptive capacity to detect events within an unrestricted "field" of awareness, without a specific focus (Raffone \& Srinivasan, 2010); this capacity can include processes of "meta- awareness" (i.e., in which practitioners are able to reflect on the process of consciousness itself).

Mindfulness - both as a meditation practice, and as a state/quality of mind - is commonly presented as an example of open-monitoring (Kabat-Zinn, 2003). However, in practice, mindfulness meditation usually involves a combination of focused attention and open-monitoring, since it usually begins with a period of focused attention on a target, such as the breath, in order to focus awareness, followed by the more receptive state of openmonitoring (Chiesa, Calati, \& Serretti, 2011).

According to Shapiro et al. (2006), the main significance of mindfulness - as a quality/state of mind, and as a meditation practice that can facilitate this - is that it involves a meta-mechanism known as "reperceiving." The three components of mindfulness 
(intention, attention and attitude) combine to generate what is described as a "fundamental shift in perspective," in which "rather than being immersed in the personal drama or narrative of our life story, we are able to stand back and witness it" (p.377). Thus, in practising mindfulness, people are seen as learning how to enter into a different relationship with their subjectivity: being able to "stand back" and dispassionately view subjective qualia as phenomena passing though their internal world, rather than identifying with and attaching to or becoming averse to such qualia (Bishop et al., 2004). This "standing back" - referred to by Shapiro et al. as "reperceiving" - is also known as "decentring," defined as "the ability to observe one's thoughts and feelings as temporary, objective events in the mind, as opposed to reflections of the self that are necessarily true" (Fresco et al., 2007, p.234).

Crucially, Shapiro et al. (2006) theorise that reperceiving/decentring has a positive impact upon wellbeing. In MBIs, the aim is not to change participants' thoughts/feelings per se, as cognitive therapy might seek to, but to help people "become more aware of, and relate differently to" this content (Shapiro, Astin, Bishop, \& Cordova, 2005, p.165). For example, Mindfulness-Based Cognitive Therapy (MBCT) is an adaptation of MBSR, designed to prevent depressive relapse (Segal, Williams, \& Teasdale, 2002). In MBCT, people are taught to decentre from their cognitions, thus helping prevent a "downward spiral" of negative thoughts and worsening negative affect which could otherwise trigger a depressive relapse. Thus MBCT, and mindfulness interventions generally, involve "retraining awareness" so that people have greater choice in how they relate and respond to their subjective experience, rather than habitually responding in maladaptive ways (Chambers, Gullone, \& Allen, 2009, p.659). The positive impact of retraining awareness in this way is not limited to depression, but extends to mental health generally. For instance, the development of decentring capabilities can help people tolerate otherwise distressing qualia, which is important given that the inability to tolerate such qualia is a transdiagnostic factor underlying diverse 
psychopathologies (Aldao, Nolen-Hoeksema, \& Schweizer, 2010), from depression (Borton, Markowitz, \& Dieterich, 2005) to substance abuse (Garland, Gaylord, Boettiger, \& Howard, 2010).

Mindfulness interventions were initially limited to clinical settings. The first such intervention was Kabat-Zinn's (1982) MBSR program, which was initially used to treat chronic pain, then was subsequently applied in the treatment of various other conditions, from cancer (Ledesma \& Kumano, 2009) to migraine (Schmidt et al., 2010). Kabat-Zinn's (1982) work was also followed by other clinical interventions which adapted the MBSR protocol for the treatment of specific mental health problems, including MBCT for the treatment of depression (Segal et al., 2002), and Mindfulness- Oriented Recovery Enhancement for the treatment of substance abuse (Garland et al., 2014).

However, since the late 1990s, there has been increasing interest in the use of mindfulness interventions in occupational contexts, not only for staff who may be suffering with stress and mental health issues, but for workers "in general" (e.g., as a protective measure against future issues). For instance, in one such early study, Shapiro, Schwartz, and Bonner (1998) reported that MBSR was effective at reducing stress among medical and pre-medical students.

Indeed, such interventions may be particularly valuable for educators, given their vulnerability to stress and other adverse work-related mental health outcomes (as discussed above). However, there have currently been no reviews assessing the impact of MBIs on the health and wellbeing of educators specifically. This is not to say that there are no summaries on the value of mindfulness in educational contexts: there have been numerous reviews into the burgeoning literature on the value of MBIs for students (e.g., Waters, Barsky, Ridd, \& Allen, 2015; Kallapiran, Koo, Kirubakaran, \& Hancock, 2015), which generally show mindfulness to be efficacious in promoting health and wellbeing, as well as outcomes such 
as academic performance. However, only two such reviews have been conducted on the use of MBIs with educators per se: one was by Albrecht, Albrecht, and Cohen (2012), which only featured three studies that had been published at that time, while a more recent report by Weare (2014) featured 13 studies. As such, to provide an updated assessment of this area, a systematic review was conducted, featuring empirical studies of the impact of mindfulness on the mental health and wellbeing of educators.

\section{Methods}

The literature search was conducted using the MEDLINE and Scopus electronic databases. The search was conducted as part of a broader systematic review on mindfulness in all spheres of occupation (which is still ongoing). The criteria for the broader review were: mindfulness AND work OR occupation OR profession OR staff (in all fields in MEDLINE and limited to article title, abstract, and keywords in Scopus). The dates selected were from the start of the database records to $10^{\text {th }}$ January 2016 . For this current review into educators, in terms of PICOS (participants, interventions, comparisons, outcomes and study design) the key criteria were: participants - currently employed in an educational context; outcomes any pertaining to mindfulness, mental health and wellbeing; and study design - any empirical study featuring data collection. Although we were principally interested in studies of MBIs in educational workplaces, as a secondary concern we were also interested in nonintervention studies on mindfulness in the workplace (e.g., regression analyses of the association between trait mindfulness and health and wellbeing outcomes). Studies were required to be published (or in press) in a peer-reviewed academic journal, and to be in English. The review was conducted according to the Preferred Reporting Items for Systematic Reviews and Meta-Analyses (PRISMA) guidelines (Moher, Liberati, Tetzlaff, \& Altman, 2009). The review protocol for the broader systematic review was registered with

the International Prospective Register of Systematic Reviews (PROSPERO) database on $5^{\text {th }}$ 
$\begin{array}{llll}\text { January } & \text { 2016. } & \text { Registration } & \text { CRD42016032899 }\end{array}$ (http://www.crd.york.ac.uk/PROSPERO).

The inclusion criteria for the broader systematic review were: 1) participants currently employed by a company or organisation; 2) empirical assessment undertaken in the context of participants' engagement with a company or organisation; 3) empirical assessment of mindfulness, mental health and wellbeing outcomes; 4) quantitative or qualitative analysis, supported by appropriate methodology; 5) published (or in press) in a peer-reviewed academic journal; and 6) written in English. Exclusion criteria were theoretical articles or commentaries without statistical or qualitative analyses. In addition to these criteria, the review in the current paper had an additional inclusion criterion namely participants currently employed in an educational context.

Papers were divided into intervention studies and non-intervention studies. For intervention studies, the following variables were extracted from each paper: type of design (e.g., RCT versus convenience sample); occupation of participants; number of experimental participants, and number of control participants (if applicable); type of MBI; length of MBI; nature of control; principal mental health and wellbeing outcomes; and the significance level of principal outcomes (for statistical analyses). For non-intervention studies, the following variables were extracted from each paper: type of analysis (e.g., regression versus qualitative); occupation of participants; number of experimental participants; principal mental health and wellbeing outcomes; and the significance level of principal outcomes (for statistical analyses).

The primary summary measures were mindfulness, mental health and wellbeing outcomes. These were principally psychometric scales pertaining to mindfulness, mental health (e.g., anger, anxiety, burnout, depression, distress, stress), wellbeing (engagement, 
satisfaction), and physical health (e.g., illness, diet, exercise, and sleep). Secondary summary measures of interest were outcomes that pertain to mental health and wellbeing (e.g., compassion, empathy, emotional intelligence and regulation, resilience, and spirituality). Finally, tertiary summary measures of interest were outcomes relating to job performance.

The Quality Assessment Tool for Quantitative Studies (QATQS; National Collaborating Centre for Methods and Tools, 2008) was used to assess the quality of the studies. QATQS assesses methodological rigor in six areas: (a) selection bias; (b) design; (c) confounders; (d) blinding; (e) data collection method; and (f) withdrawals and drop-outs. Each area is assessed on a quality score of one to three (one $=$ strong; two = moderate; three = weak). Scores for each area were collated, and a global score was assigned to each study. If there are no weak ratings, the study is given a score of one (judged as strong); one weak rating leads to a score of two (moderate); and two or more weak ratings generates a score of three (weak) (Supplementary Materials). QATQS scoring was conducted (II) and checked independently (TL). Any discrepancies were resolved by discussion with agreement reached in all cases.

\section{Results}

\section{Search Results.}

For the broader systematic review (i.e., mindfulness across all occupations), following removal of duplicate citations, 722 potentially relevant papers were identified. In the current specific systematic review (focusing specifically on educators), from reviewing the abstract, 606 papers were excluded. 
Table 1. Overview of Intervention Studies.

\begin{tabular}{|c|c|c|c|c|c|c|c|c|}
\hline Authors & Occupation & Design & $\begin{array}{l}\text { Expt. } \\
\text { group }\end{array}$ & $\begin{array}{l}\text { Control } \\
\text { group }\end{array}$ & Intervention & Length & Control & Primary outcome(s) \\
\hline $\begin{array}{l}\text { Baccarani et al. } \\
\text { (2013) }\end{array}$ & $\begin{array}{l}\text { University } \\
\text { administrators }\end{array}$ & RCT & 10 & 10 & $\begin{array}{l}\text { Mindfulness program } \\
\text { (specific to study) }\end{array}$ & 4 weeks & NR & $\begin{array}{l}\text { PI }>\text { general wellbeing }(p=.002) \& \text { selective attention }(p= \\
.011)\end{array}$ \\
\hline $\begin{array}{l}\text { Beshai et al. } \\
\text { (2015) }\end{array}$ & Teachers & $\begin{array}{l}\text { Convenienc } \\
\text { e sample }\end{array}$ & 49 & 40 & .b Foundations course & $\begin{array}{l}9 \\
\text { session }\end{array}$ & Wait-list & $\begin{array}{l}\mathrm{PI}<\text { stress }(p<.01) . \mathrm{PI}>\text { compassion }(p<.01) \\
\text { mindfulness }(p<.01), \& \text { wellbeing }(p<.01)\end{array}$ \\
\hline $\begin{array}{l}\text { Flook et al. } \\
(2013)\end{array}$ & Teachers & $\mathrm{RCT}$ & 9 & 9 & MBSR adaptation & 8 weeks & Wait-list & $\begin{array}{l}\text { PI }<\text { burnout }(p<.05) \& \text { distress }(p<.001) . \mathrm{PI}>\text { attention } \\
(p<.05) \& \text { mindfulness }(p<.05) .\end{array}$ \\
\hline $\begin{array}{l}\text { Franco et al. } \\
(2010)\end{array}$ & Teachers & RCT & 34 & 34 & $\begin{array}{l}\text { Mindfulness program } \\
\text { (specific to study) }\end{array}$ & $\begin{array}{l}10 \\
\text { weeks }\end{array}$ & $\begin{array}{l}\text { Music } \\
\text { listening }\end{array}$ & $\begin{array}{l}\text { PI }<\text { anxiety }(p=.008), \text { depression }(p=.001), \& \text { distress }(p \\
=.001) .\end{array}$ \\
\hline $\begin{array}{l}\text { Frank, Riebel, et } \\
\text { al. (2015) }\end{array}$ & Teachers & $\mathrm{RCT}$ & 18 & 18 & MBSR & 8 weeks & Wait-list & $\begin{array}{l}\text { PI }>\text { self-regulation }(p=.003) \text {, calmness }(p=.002) \text {, } \\
\text { mindfulness }(p=.01) \text {, self-compassion }(p=.003) \text {, sleep } \\
\text { duration }(p=.01) \& \text { sleep quality }(p=.001) . \text { PI }><\text { anxiety, } \\
\text { burnout, depression. }\end{array}$ \\
\hline Gold et al. (2010) & $\begin{array}{l}\text { Teachers (9) } \\
\text { and assistants } \\
(2)\end{array}$ & $\begin{array}{l}\text { Convenienc } \\
\text { e sample }\end{array}$ & 11 & - & MBSR & 8 weeks & N/A & $\begin{array}{l}\mathrm{PI}<\text { depression }(p<.02) \text {, stress }(p<.05) . \mathrm{PI}><\text { anxiety } \& \\
\text { mindfulness. }\end{array}$ \\
\hline $\begin{array}{l}\text { Harris et al. } \\
(2015)\end{array}$ & Teachers & $\mathrm{RCT}$ & 34 & 30 & CALM & $\begin{array}{l}16 \\
\text { weeks }\end{array}$ & Wait-list & $\begin{array}{l}\mathrm{PI}>\text { distress tolerance }(p<.01) \text {, health }(p<.05) \text {, } \\
\text { mindfulness }(p<.05) \& \text { positive affect }(p<.01) \text {. PI }>< \\
\text { burnout or sleep quality. }\end{array}$ \\
\hline $\begin{array}{l}\text { Hue and Lau } \\
\text { (2015) }\end{array}$ & Trainee teachers & $\begin{array}{l}\text { Convenienc } \\
\text { e sample }\end{array}$ & $35(78)$ & 35 & $\begin{array}{l}\text { Mindfulness program } \\
\text { (specific to study) }\end{array}$ & 6 weeks & Nothing & $\begin{array}{l}\text { PI }>\text { mindfulness }(p=.023) \& \text { wellbeing }(p=.022) . \text { PI }>< \\
\text { anxiety, depression \& stress. }\end{array}$ \\
\hline $\begin{array}{l}\text { Jennings et al. } \\
\text { (2011) }\end{array}$ & Teachers & $\begin{array}{l}\text { Convenienc } \\
\text { e sample }\end{array}$ & $\begin{array}{l}31(1) \& \\
43(2)\end{array}$ & - & $\begin{array}{l}\text { Cultivating awareness } \\
\& \text { resilience in } \\
\text { education } * *\end{array}$ & $\begin{array}{l}1 \text { month } \\
(2 \\
\text { w'end })\end{array}$ & N/A & $\begin{array}{l}\text { PI }><\text { depression, mindfulness, negative affect, positive } \\
\text { affect, self-efficacy, \& time pressure. }\end{array}$ \\
\hline $\begin{array}{l}\text { Jennings et al. } \\
\text { (2013) }\end{array}$ & Teachers & $\mathrm{RCT}$ & 25 & 25 & $\begin{array}{l}\text { Cultivating awareness } \\
\& \text { resilience in } \\
\text { education } * *\end{array}$ & $\begin{array}{l}1 \text { month } \\
(2 \\
\text { w'end })\end{array}$ & Wait-list & $\begin{array}{l}\mathrm{PI}<\text { time pressure }(p=.025) . \mathrm{PI}>\text { health }(p=.004) \\
\text { mindfulness }(p=.003), \& \text { self-efficacy }(p=.002) . \mathrm{PI}>< \\
\text { negative affect, positive affect. }\end{array}$ \\
\hline Klatt et al. (2009) & $\begin{array}{l}\text { University } \\
\text { employees }\end{array}$ & $\mathrm{RCT}$ & 22 & 20 & MBSR adaptation & & & $\begin{array}{l}\mathrm{PI}<\text { stress }(p=.002) . \mathrm{PI}>\text { mindfulness }(p=.014) \text {, sleep } \\
\text { quality }(p=.016) .\end{array}$ \\
\hline $\begin{array}{l}\text { Malarkey et al. } \\
\text { (2013) }\end{array}$ & $\begin{array}{l}\text { University } \\
\text { employees }\end{array}$ & $\mathrm{RCT}$ & 93 & 93 & $\begin{array}{l}\text { Mindfulness program } \\
\text { (specific to study) }\end{array}$ & 8 weeks & $\begin{array}{l}\text { Lifestyle } \\
\text { education } \\
\text { programm } \\
\text { e }\end{array}$ & $\begin{array}{l}\text { PI }>\text { mindfulness }(p=.003) \text {. PI }><\text { depression, sleep quality } \\
\& \text { stress. }\end{array}$ \\
\hline $\begin{array}{l}\text { Poulin et } \\
\text { al. (2008) }\end{array}$ & Teachers & $\mathrm{RCT}$ & 28 & 16 & $\begin{array}{l}\text { Mindfulness-based } \\
\text { wellbeing } \\
\text { education }\end{array}$ & 8 weeks & Nothing & $\begin{array}{l}\text { PI }>\text { mindfulness }(p<.001) \text {, satisfaction with life }(p<.05) \text {, } \\
\text { self-efficacy }(p<.05) ., \& \text { self-rated health }(p<.05) . \text { PI }>< \\
\text { distress }\end{array}$ \\
\hline
\end{tabular}




\begin{tabular}{|c|c|c|c|c|c|c|c|c|}
\hline $\begin{array}{l}\text { Ramsey and } \\
\text { Jones }\end{array}$ & Teachers & $\mathrm{RCT}$ & $13(22)$ & $24(29)$ & $\begin{array}{l}\text { Mindfulness } \\
\text { workshop (specific to }\end{array}$ & 1 day & NR & $\begin{array}{l}\text { PI }>\text { relationships [perceived instigated ostracism] }(p= \\
.014) \text {. }\end{array}$ \\
\hline $\begin{array}{l}\text { Roeser et } \\
\text { al. (2013) }\end{array}$ & Teachers & $\mathrm{RCT}$ & 54 & 59 & Mindfulness Training & 8 weeks & Wait-list & $\begin{array}{l}\text { PI }<\text { anxiety }(p<.01) \text {, burnout }(p<.01) \text {, depression }(p<\text { s } 1 \text {, stress }(p<.01) . \text { PI }>\text { self-compassion }(p<.01) \\
\& \text { mindfulness }(p<.01) \text {. PI }><\text { blood pressure. }\end{array}$ \\
\hline $\begin{array}{l}\text { Schussler et } \\
\text { al. (2015) }\end{array}$ & Teachers & $\begin{array}{l}\text { Conven } \\
\text { ienc e }\end{array}$ & 50 & - & CARE & 8 weeks & N/A & Qualitative focus groups. PI > self-regulation \\
\hline Taylor et al. & Teachers & $\mathrm{RCT}$ & 26 & 30 & SMART & 8 weeks & Wait-list & $\mathrm{PI}<$ stress $(p<.001) . \mathrm{PI}><$ compassion. \\
\hline
\end{tabular}

Note. $\langle=$ decreases in $;>=$ increases in $;\rangle=$ no change in $: !=$ mindfulness associated with worsened outcome; expt $=$ experimental group $\cdot$ cnt $=$ control group $\cdot$ PI $=$ post-intervention $N R$

$=$ not-reported; $M B C T=$ mindfulness-based cognitive therapy; $M B S R=$ mindfulness-based stress reduction; $M B S T=$ mindfulness-based stress reduction therapy. $C A L M=$ community approach to learning mindfully. $\mathrm{CARE}=$ cultivating awareness and resilience in education. $S M A R T=$ stress management and relaxation training. $M M=$ mindfulness meditation; $N C C=$ neural correlates of consciousness; $N R=$ not recorded; $N / A=$ not applicable; $N A=$ not available; $R C T=$ randomized controlled trial; $* *$ number in parenthesis is the initial sample size (if different from sample size featured in analysis); $* *=$ mindfulness just one component of broader intervention

\section{Table 2. Overview of non-intervention studies}

\begin{tabular}{|c|c|c|c|c|c|}
\hline Authors & Workplace & $\begin{array}{l}\text { Meditator } \\
\text { S }\end{array}$ & $\begin{array}{l}\text { Non- } \\
\text { meditators }\end{array}$ & Analysis & Primary result \\
\hline $\begin{array}{l}\text { Frank, Jennings et } \\
\text { al. (2015) }\end{array}$ & Teachers & - & $\begin{array}{l}918(263,263, \\
392)\end{array}$ & Regression & $\begin{array}{l}\text { Mindfulness correlation: < burnout }(p<.01) .>\text { behaviour management } \\
\text { efficacy }(p<.01) .\end{array}$ \\
\hline Jennings (2015) & $\begin{array}{l}\text { Early childhood } \\
\text { teachers }\end{array}$ & - & 35 & & $\begin{array}{l}\text { Mindfulness correlation: }>\text { emotional support }(p<.001) .><\text { classroom } \\
\text { organisation \& instructional support. }\end{array}$ \\
\hline
\end{tabular}

Note. $<=$ negative correlation with $>=$ positive correlation with $;><=$ no correlation; 


\section{Relationship between Mindfulness and Key Outcomes}

An overview of the findings is shown in table 3 below. This shows whether outcomes were either: (a) increased in relation to an MBI; (b) did not change in relation to an MBI (or in exceptional cases, changed in a "negative" direction); or (c) were found in non-intervention studies to be associated with mindfulness (i.e., through regression analyses). A more detailed presentation of the results is then shown in table 4 below; this lists all the specific assessment tools used for each measure, together with the specific studies deploying that tool.

Table 3. Summary of common outcomes across all studies

\begin{tabular}{lllll}
\hline Outcome & $\begin{array}{l}\text { Number of } \\
\text { studies }\end{array}$ & $\begin{array}{l}\text { Improvement related to } \\
\text { mindfulness intervention }\end{array}$ & $\begin{array}{l}\text { No change in relation to } \\
\text { mindfulness }\end{array}$ & $\begin{array}{l}\text { Association } \\
\text { (benign) with }\end{array}$ \\
\hline Anxiety & 3 & 2 & 1 & 0 \\
Burnout \& & 7 & 4 & 2 & 1 \\
Compassion \& & 4 & 3 & 1 & 0 \\
Depression & 4 & 3 & 1 & 0 \\
Distress \& anger & 5 & 3 & 2 & 0 \\
Emotional & 3 & 3 & 0 & 0 \\
Health & 8 & 5 & 5 & 0 \\
Job performance & 4 & 2 & 0 & 3 \\
Mindfulness \& & 14 & 12 & 2 & 0 \\
Stress \& strain & 6 & 4 & 2 & 0 \\
Wellbeing \& & 6 & 5 & 2 & 0 \\
\hline
\end{tabular}


Table 4. Common outcomes across all studies

\begin{tabular}{|c|c|c|c|c|}
\hline Outcome & Measure & $\begin{array}{l}\text { Improvement (positive change) related to } \\
\text { mindfulness intervention }\end{array}$ & $\begin{array}{l}\text { No change in relation to } \\
\text { mindfulness intervention }\end{array}$ & Association (benign) with mindfulness \\
\hline \multirow[t]{2}{*}{ Anxiety } & State trait anxiety inventory & Johnson et al. (2015), Roeser et al. (2013) & & \\
\hline & Symptom checklist-90-R [anxiety] & Franco et al. (2010) & & \\
\hline \multirow[t]{3}{*}{ Burnout } & Connor David resiliency scale & Klatt et al. (2015) & & \\
\hline & Maslach burnout inventory & Flook et al. (2013), Roeser et al. (2013) & $\begin{array}{l}\text { Frank, Riebel. et al. (2015), } \\
\text { Harris et al. (2015) }\end{array}$ & Frank, Jennings, et al. (2015) \\
\hline & $\begin{array}{l}\text { Utrecht work engagement scale } \\
\text { [vigour] }\end{array}$ & Klatt et al. (2015) & & \\
\hline \multirow{2}{*}{$\begin{array}{l}\text { Empathy \& } \\
\text { compassion }\end{array}$} & Santa Clara brief compassion scale & & Taylor et al. (2015) & \\
\hline & Self-compassion scale & $\begin{array}{l}\text { Beshai et al. (2015), Frank, Riebel. et al. (2015) } \\
\text { Roeser et al. (2013) }\end{array}$ & & \\
\hline \multirow[t]{3}{*}{ Depression } & Beck depression inventory & Roeser et al. (2013)) & & \\
\hline & Brief symptom inventory & & Frank, Riebel. et al. (2015) & \\
\hline & $\begin{array}{l}\text { Symptom checklist- } 90-\mathrm{R} \\
\text { [depression] }\end{array}$ & Franco et al. (2010) & & \\
\hline \multirow{4}{*}{$\begin{array}{l}\text { Distress \& } \\
\text { anger }\end{array}$} & Brief symptom inventory & & Frank, Riebel. et al. (2015) & \\
\hline & Distress tolerance scale & Harris et al. (2015) & & \\
\hline & $\begin{array}{l}\text { Kessler } 10 \text { psychological distress } \\
\text { scale }\end{array}$ & & Poulin et al. (2008) & \\
\hline & Symptom checklist-90-R & Flook et al. (2013), Franco et al. (2010) & & \\
\hline \multirow{3}{*}{$\begin{array}{l}\text { Emotional } \\
\text { intelligence \& } \\
\text { regulation }\end{array}$} & $\begin{array}{l}\text { Affective self-regulatory efficacy } \\
\text { scale }\end{array}$ & Frank, Riebel. et al. (2015) & & \\
\hline & Emotion regulation questionnaire & Jennings et al. (2013) & & \\
\hline & Qualitative interviews & Schussler et al. (2015) & & \\
\hline \multirow[t]{8}{*}{ Health } & Blood pressure & & Roeser et al. (2013) & \\
\hline & Daily physical symptoms scale & Harris et al. (2015), Jennings et al. (2013) & Jennings et al. (2011) & \\
\hline & Interleukin 6 & & Malarkey et al.( 2013)！ & \\
\hline & $\begin{array}{l}\text { Perceived stress scale [sleep } \\
\text { duration] }\end{array}$ & & Klatt et al. (2009) & \\
\hline & $\begin{array}{l}\text { Perceived stress scale [sleep } \\
\text { quality] }\end{array}$ & Klatt et al. (2009) & & \\
\hline & Pittsburgh sleep quality index & Frank, Riebel. et al. (2015) & & \\
\hline & Self-rated health & Poulin et al. (2008) & & \\
\hline & Sleep-related impairment scale & & Harris et al. (2015) & \\
\hline
\end{tabular}




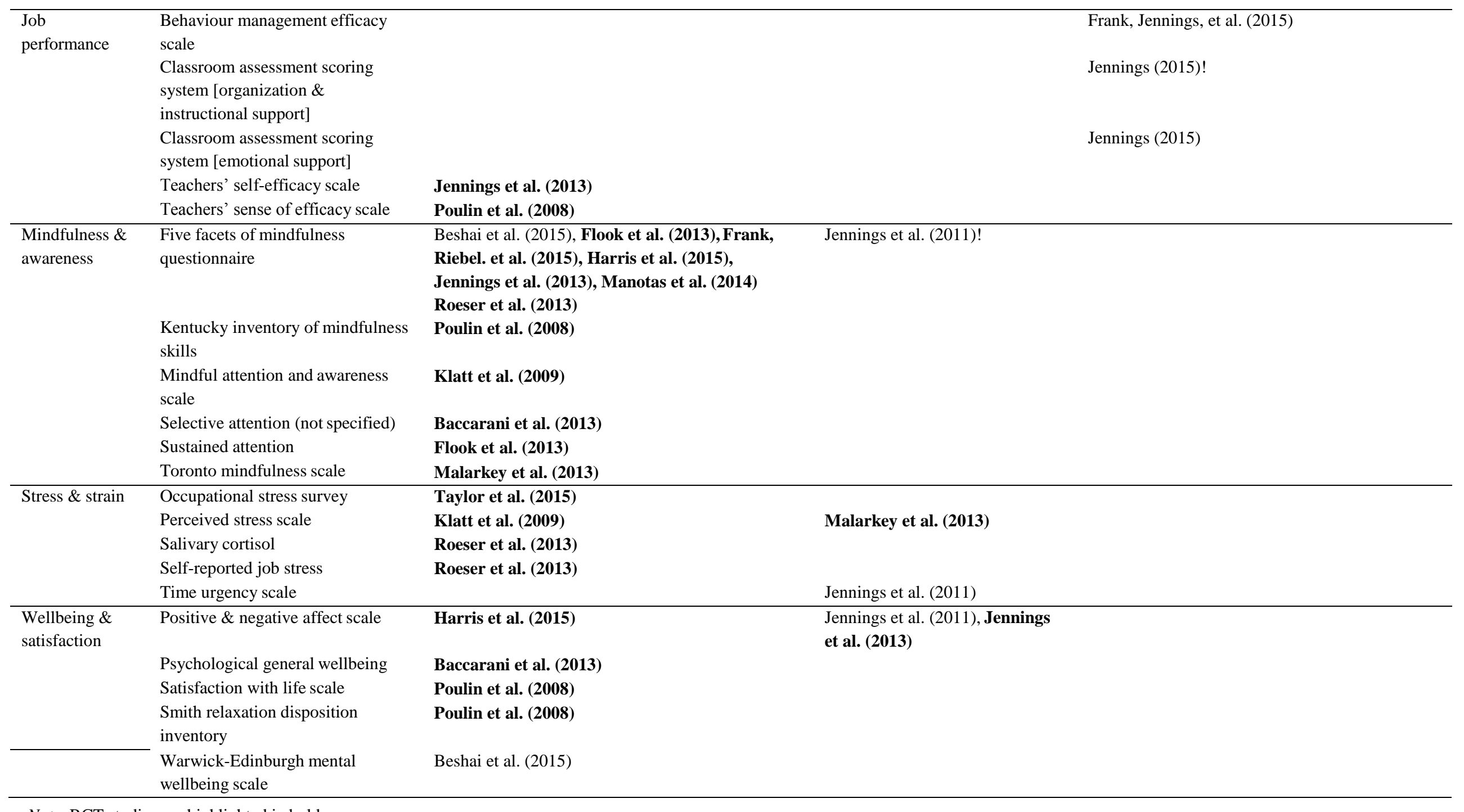

Note. RCT studies are highlighted in bold. 


\section{Discussion}

The main finding to emerge from the systematic review is that MBIs overwhelmingly had a positive impact upon all outcome measures, with the exception of burnout (where the findings were more equivocal). Thus, overall, the review corroborated the positive appraisal of the value of mindfulness for educators provided by Albrecht et al. (2012) and Weare (2014). Before dealing with the various outcomes in turn, we can begin by observing that the MBIs certainly appeared effective at facilitating the development of mindfulness, which was assessed by 14 intervention studies: of these, the vast majority found increased mindfulness in relation to the MBI $(n=12)$, with only two finding no increase. It is interesting to note that a range of different psychometric scales $(n=10)$ were deployed across the studies, which is perhaps both a weakness and a strength. It is a weakness inasmuch as the lack of a dominant standardised scale makes it difficult to draw comparisons across studies, and to aggregate the findings through meta-analyses. The latter is particularly important in terms of trying to draw any more substantive conclusions around the value of mindfulness. This inconsistency in the use of scales across different studies was a common theme in this review, and is something that mindfulness scholars may wish to address going forward (as discussed further below).

That said, the diversity of measures does allow us to discern nuances in the development of mindfulness. The most popular tool, used in eight studies, was Baer, Smith, Hopkins, Krietemeyer, and Toney's (2006) 39-item Five Facets of Mindfulness Scale. This widely used tool (with 2,171 citations as of January 2016) features five different dimensions/skills: describing, acting with awareness, non-judging of inner experience, and non-reactivity to inner experience. In contrast, Brown and Ryan's (2003) Mindful Attention and Awareness Scale is arguably more prevalent in the literature (with 4,127 citations as of January 2016), but featured in only one study here. This assesses dispositional mindfulness, gauging "individual differences in the frequency of mindful states over time" (p.824). It focuses on a single, core characteristic of mindfulness, namely open and receptive awareness, which essentially aligns with Kabat-Zinn's (2003) definition cited above. Clearly, this complements the multidimensionality of Brown and Ryan's (2003) scale, and 
in future we would recommend that studies use both tools.

Turning to the specific outcomes, on balance mindfulness appears to have a beneficial impact upon most metrics of mental health, although the results were by no means unequivocal. For instance, with burnout, while three studies found that this was reduced in relation to an MBI (Flook, Goldberg, Pinger, Bonus, \& Davidson, 2013; Klatt, Steinberg, \& Duchemin, 2015; Roeser et al., 2013), two found no significant changes (Frank, Reibel, Broderick, Cantrell, \& Metz, 2015; Harris, Jennings, Katz, Abenavoli, \& Greenberg, 2015), although in the latter two studies the results were certainly close to significance in the expected direction. Similarly, with depression, while three studies found that an MBI significantly reduced this (Franco, Mañas, Cangas, Moreno, \& Gallego, 2010; Gold et al., 2010; Roeser et al., 2013), Frank et al. (2015) found no significant change (although the results were again approaching significance). With stress, four studies observed a reduction in connection with an MBI (Gold et al., 2010; Klatt et al., 2015; Roeser et al., 2013; Taylor et al., 2015), while two found no significant change (Jennings, Snowberg, Coccia, \& Greenberg, 2011; Malarkey, Jarjoura, \& Klatt, 2013).

Away from mental health per se, mindfulness was also associated with wellbeing generally, with four studies finding MBIs significantly increasing wellbeing/satisfaction (Baccarani, Mascherpa, \& Minozzo, 2013; Beshai, McAlpine, Weare, \& Kuyken, 2015; Harris et al., 2015; Poulin, Mackenzie, Soloway, \& Karayolas, 2008), while two found no significant changes (Jennings et al., 2011; Jennings, Frank, Snowberg, Coccia, \& Greenberg, 2013). The positive impact of MBIs spanned outcomes including positive affect (Harris et al., 2015), relaxation (Poulin et al., 2008), satisfaction with life (Poulin et al., 2008), and psychological wellbeing satisfaction (Baccarani et al., 2013; Beshai et al., 2015). The findings for health were rather more equivocal, with five studies finding significant improvements in health relating to an MBI (Frank et al., 2015; Klatt, Buckworth, \& Malarkey, 2009; Harris et al., 2015; Jennings et al., 2013; Poulin et al., 2008), but a further five finding no significant changes (Klatt et al., 2009; Harris et al., 2015; Jennings et al., 2011; Roeser et al., 2013; Malarkey et al., 2013). With health, the positive changes included reduced daily physical 
symptoms (Harris et al., 2015; Jennings et al., 2011) and improved sleep (Klatt et al., 2009). However, no changes were observed in relation to blood pressure (Roeser et al., 2013), while Malarkey et al. (2013) found that inflammation - as indexed by Interleukin 6, an endogenous chemical active in inflammation - actually worsened in relation to an MBI.

In addition to these primary wellbeing outcomes, mindfulness was also linked to various skills and qualities that are associated with wellbeing, and which may help to provide an explanation for the generally positive outcomes adumbrated above. For instance, three studies examined the relationship between mindfulness and emotional regulation, with all three suggesting that MBIs significantly increased emotional regulation (Frank et al., 2015; Jennings et al., 2013; Schussler, Jennings, Sharp, \& Frank, 2015). As outlined above, according to Shapiro et al. (2006), the key mechanism through which mindfulness exerts its positive effects is that of "reperceiving," also known as decentring (Fresco et al., 2007). This ability means that people are better able to detach themselves from distressing qualia that might otherwise precipitate feelings of stress etc. More generally, reperceiving could be regarded as an aspect of a more general capacity of emotion regulation. For instance, Walsh and Shapiro (2006) define meditation as "a family of self-regulation practices that focus on training attention and awareness in order to bring mental processes under greater voluntary control and thereby foster general mental well-being” (pp.228-229). Thus, the suggestion is that mindfulness might positively impact on wellbeing in the following way: (a) mindfulness involves introspective practices that facilitate the development of attention and awareness skills; (b) the development of these skills leads to enhanced emotional regulation and intelligence (including abilities such as reperceiving); and (c) emotional regulation and intelligence are meta-skills that subserve multiple health and wellbeing outcomes (while, conversely, poor emotion regulation skills are a transdiagnostic factor underlying diverse psychopathologies; Aldao et al., 2010). Future work may help to elucidate these hypothesised causal chains further, e.g., through longitudinal studies deploying regression analyses.

Finally, the impact of mindfulness was not limited to the mental health and wellbeing of 
employees but also was associated with enhanced job performance, although this was only assessed by a handful of studies. Both Jennings et al. (2013) and Poulin et al. (2008) found that MBIs enhanced teachers' sense of self-efficacy, while non-interventions studies found that mindfulness was associated with outcomes such as behaviour and classroom management (Frank, Jennings, \& Greenberg, 2015; Jennings, 2015).

\section{Conclusions and Recommendations}

On the whole, the results are relatively encouraging. MBIs did appear to have a largely positive impact on the mental health and wellbeing of educators. With respect to all outcomes, the majority of studies reported statistically-significant improvements. In terms of mental health outcomes, the findings included positive results for anxiety (two out of three studies finding an improvement), burnout and resilience (four out of seven), depression (three out of four), distress and anger (three out of five), and stress and strain (four out of six). With respect to wellbeing outcomes more broadly, the findings included positive results for mindfulness (12 out of 14), compassion and empathy (three out of four), emotional regulation (three out of three), wellbeing and satisfaction (five out of six), health (five out of eight), and job performance (three out of four).

These positive conclusions must be tempered by a number of caveats. Firstly, the quality of the studies was relatively poor. According to the QATQS scoring protocol, the majority of the studies only achieved a global rating of "weak", due to factors such as poor monitoring of attrition and insufficient attention to confounders. Obviously, future research will hopefully remedy these flaws, enabling a stronger and more reliable research base to be built. Secondly, the research is currently largely biased towards interventions that were developed for use in clinical settings, and relatedly, the assessments tend to mostly use metrics pertaining to mental health. While such interventions and metrics are of course valuable, it would be good in future to see interventions and outcomes that are also geared towards more 'positive' wellbeing constructs, such as work engagement (Schaufeli \& Bakker, 2003). As a final point, it is also important not to regard MBIs as any kind of panacea for stress, nor as a sustainable remedy for an education system that imposes 
such stressors to begin with. It is encouraging that MBIs are helpful to educators, but the encouragement and implementation of such interventions must not come at the expense of trying to create a system that is less inherently stressful. Indeed, this is a broader concern regarding the use of mindfulness in occupational contexts, where some scholars are raising concerns about MBIs being used to help workers "adapt" to a toxic work environment, as opposed to employers striving to reduce the toxicity of the work itself (Van Gordon, Shonin, Zangeneh, \& Griffiths, 2014). Nevertheless, all that being said, while educators are subjected to these kinds of work-related burdens, then it would appear that MBIs can be of assistance in enabling them to cope.

To conclude, based on the above considerations, we have a number of recommendations regarding the future implementation and assessment of MBIs in the context of teaching and teacher training. Let's take implementation first. To begin with, given the largely promising results above, it would be ideal to see MBIs being offered in all teacher training courses and in all educational environments. That is, ideally all educators would be given the opportunity to attend at least one MBI, e.g., lasting eight weeks. If resources permit, courses and educational settings could also include provisions for on-going practice (e.g., weekly drop-in sessions). However, if resources did not allow that, the introductory MBI would at least introduce mindfulness to educators, who would then have the opportunity to pursue this on their own time (e.g., in the community). Of course, the caveat above still holds about such interventions not being used to mask a toxic work environment, nor placing the onus on staff to simply be 'resilient' to these. In addition, it is vital that participation not be compulsory. While many participants may well benefit, mindfulness may not be to everyone's taste, or within their 'comfort zone' (see e.g., Author et al., 2015b). More seriously, it may be even harmful to people with certain pre-existing or current mental health conditions (see e.g., Dobkin, Irving, \& Amar, 2012). As such, a degree of sensitivity will be necessary in terms of encouraging and facilitating participation.

As to which MBIs might be offered, this is an interesting question. On the one hand, there are good arguments for using MBIs that have been well-tested and validated, such as MBCT and 
MBSR. At the same time though, such MBIs were created primarily for clinical populations. There is thus also an argument for the development of new programmes suited specifically to certain contexts, as we have seen with the creation of bespoke MBIs suited to schoolchildren (Waters et al., 2015). As such, there is certainly room for the development of MBIs particularly suited to educators, as indeed Malarkey et al. (2013) have done. Similarly, such programmes may not only want to focus on a 'deficit model' of mental health (e.g., reducing outcomes like anxiety), but may also be able to aim towards more positive wellbeing outcomes, such as work engagement (Schaufeli \& Bakker, 2003).

Of course, introducing new initiatives carries its own issues, most notably a lack of empirical validation. As such, the future implementation of MBIs in educational contexts - including the careful development and introduction of new MBIs - will ideally be accompanied by a concomitant program of empirical assessment. With such assessment, researchers should obviously aim for best practice in this regard, like the use of randomised controlled trials (RCTs) with adequate sample sizes. (In fact, the existing literature is already quite good in this respect, with 12 of the 17 intervention trials analysed here employing an RCT design.) In addition, researchers might also consider broadening their assessment repertoire, not only analysing deficit-based mental health outcomes (e.g., anxiety, stress), but also more positive wellbeing-related outcomes, such as engagement (Schaufeli \& Bakker, 2003). In this way, over time, we may be able to build up an even clearer understanding of the potential value of mindfulness for educators.

\section{References}

Albrecht, N., Albrecht, P., \& Cohen, M. (2012). Mindfully teaching in the classroom: A literature review. Australian Journal of Teacher Education, 37(12), 1-14. doi: 10.14221/ajte.2012v37n12.2

Aldao, A., Nolen-Hoeksema, S., \& Schweizer, S. (2010). Emotion-regulation strategies across psychopathology: A meta-analytic review. Clinical Psychology Review, 30(2), 217-237. doi: 10.1016/j.cpr.2009.11.004

Baccarani, C., Mascherpa, V., \& Minozzo, M. (2013). Zen and well-being at the workplace. TQM Journal, 25(6), 606-624. doi: 10.1108/TQM-07-2013-0077 
Baer, R. A., Smith, G. T., Hopkins, J., Krietemeyer, J., \& Toney, L. (2006). Using self-report assessment methods to explore facets of mindfulness. Assessment, 13(1), 27-45. doi: $10.1177 / 1073191105283504$

Bearse, J. L., McMinn, M. R., Seegobin, W., \& Free, K. (2013). Barriers to psychologists seeking mental health care. Professional Psychology: Research and Practice, 44(3), 150-157. doi: $10.1037 / \mathrm{a} 0031182$

Beshai, S., McAlpine, L., Weare, K., \& Kuyken, W. (2015). A non-randomised feasibility trial assessing the efficacy of a mindfulness-based intervention for teachers to reduce stress and improve well-being. Mindfulness, 7(1), 198-208. doi: 10.1007/s12671-015-0436-1

Bishop, S. R., Lau, M., Shapiro, S., Carlson, L., Anderson, N. D., Carmody, J., . . Devins, G. (2004). Mindfulness: A proposed operational definition. Clinical Psychology: Science and Practice, 11(3), 230-241. doi: 10.1093/clipsy.bph077

Blomberg, S., \& Knight, B. A. (2015). Investigating novice teacher experiences of the teaching dynamics operating in selected school communities in Finland. Improving Schools, 18(2), 157-170. doi: 10.1177/1365480215576176

Borton, J. L. S., Markowitz, L. J., \& Dieterich, J. (2005). Effects of suppressing negative self-referent thoughts on mood and self-esteem. Journal of Social and Clinical Psychology, 24(2), 172190. doi: 10.1521/jscp.24.2.172.62269

Brown, K. W., \& Ryan, R. M. (2003). The benefits of being present: Mindfulness and its role in psychological well-being. Journal of Personality and Social Psychology, 84(4), 822-848. doi: $10.1037 / 0022-3514.84 .4 .822$

Chambers, R., Gullone, E., \& Allen, N. B. (2009). Mindful emotion regulation: An integrative review.Clinical Psychology Review, 29(6), 560-572. doi: 10.1016/j.cpr.2009.06.005

Chew-Graham, C. A., Rogers, A., \& Yassin, N. (2003). "I wouldn"t want it on my CV or their records": Medical students' experiences of help-seeking for mental health problems. Medical Education, 37(10), 873-880. doi: 10.1046/j.1365-2923.2003.01627.x

Chiesa, A., Calati, R., \& Serretti, A. (2011). Does mindfulness training improve cognitive abilities? A systematic review of neuropsychological findings. Clinical Psychology Review, 31(3), 449- 464. doi: 10.1016/j.cpr.2010.11.003

Cooke, P. J., Melchert, T. P., \& Connor, K. (2016). Measuring well-being: A review of instruments.The Counseling Psychologist, 44(5), 730-757. doi: 10.1177/0011000016633507

Cousins, L. S. (1996). The dating of the historical Buddha: A review article. Journal of the Royal Asiatic Society (Third Series), 6(1), 57-63. doi: doi:10.1017/S1356186300014760

Davies, S. (2014). Annual Report of the Chief Medical Officer 2013, Public Mental Health Priorities: 
Investing in the Evidence. London: Department of Health.

de Chavez, A. C., Backett-Milburn, K., Parry, O., \& Platt, S. (2005). Understanding and researching wellbeing: Its usage in different disciplines and potential for health research and health promotion. Health Education Journal, 64(1), 70-87. doi: 10.1177/001789690506400108

Diener, E. (2000). Subjective well-being: The science of happiness and a proposal for a national index. American Psychologist, 55(1), 34-43. doi: 10.1037/0003-066X.55.1.34

Dobkin, P. L., Irving, J. A., \& Amar, S. (2012). For whom may participation in a mindfulness-based stress reduction program be contraindicated?. Mindfulness, 3(1), 44-50. doi: 10.1007/s12671-011-0079-9

Engel, G. L. (1977). The need for a new medical model: A challenge for biomedicine. Science, 196(4286), 129-136. doi: 10.1126/science.847460

Flook, L., Goldberg, S. B., Pinger, L., Bonus, K., \& Davidson, R. J. (2013). Mindfulness for teachers: A pilot study to assess effects on stress, burnout, and teaching efficacy. Mind, Brain, and Education, 7(3), 182-195. doi: 10.1111/mbe.12026

Franco, C., Mañas, I., Cangas, A. J., Moreno, E., \& Gallego, J. (2010). Reducing teachers' psychological distress through a mindfulness training program. Spanish Journal of Psychology, 13(2), 655-666. doi: 10.1017/S1138741600002328

Frank, J. L., Jennings, P. A., \& Greenberg, M. T. (2015). Validation of the Mindfulness in Teaching Scale. Mindfulness, 7(1), 155-163. doi: 10.1007/s12671-015-0461-0

Frank, J. L., Reibel, D., Broderick, P., Cantrell, T., \& Metz, S. (2015). The effectiveness of Mindfulness-Based Stress Reduction on educator stress and well-being: Results from a pilot study. Mindfulness, 6(2), 208-216. doi: 10.1007/s12671-013-0246-2

Fresco, D. M., Moore, M. T., van Dulmen, M. H. M., Segal, Z. V., Ma, S. H., Teasdale, J. D., \& Williams, J. M. G. (2007). Initial psychometric properties of the experiences questionnaire: Validation of a self-report measure of decentering. Behavior Therapy, 38(3), 234-246. doi: 10.1016/j.beth.2006.08.003

Frisch, M. B., Cornell, J., Villanueva, M., \& Retzlaff, P. J. (1992). Clinical validation of the Quality of Life Inventory. A measure of life satisfaction for use in treatment planning and outcome assessment. Psychological Assessment, 4(1), 92-101. doi: 10.1037/1040-3590.4.1.92

Garland, E. L., Gaylord, S. A., Boettiger, C. A., \& Howard, M. O. (2010). Mindfulness training modifies cognitive, affective, and physiological mechanisms implicated in alcohol dependence: Results of a randomized controlled pilot trial. Journal of Psychoactive Drugs, 42(2), 177-192. doi: 10.1080/02791072.2010.10400690

Garland, E. L., Manusov, E. G., Froeliger, B., Kelly, A., Williams, J. M., \& Howard, M. O. (2014). Mindfulness-oriented recovery enhancement for chronic pain and prescription opioid 
misuse: Results from an early-stage randomized controlled trial. Journal of Consulting and Clinical Psychology, 82(3), 448-459. doi: 10.1037/a0035798

George, D. R., Dellasega, C., Whitehead, M. M., \& Bordon, A. (2013). Facebook-based stress management resources for first-year medical students: A multi-method evaluation. Computers in Human Behavior, 29(3), 559-562. doi: 10.1016/j.chb.2012.12.008

Gold, E., Smith, A., Hopper, I., Herne, D., Tansey, G., \& Hulland, C. (2010). Mindfulness-based stress reduction (MBSR) for primary school teachers. Journal of Child and Family Studies, 19(2), 184-189. doi: 10.1007/s10826-009-9344-0

Harris, A. R., Jennings, P. A., Katz, D. A., Abenavoli, R. M., \& Greenberg, M. T. (2015). Promoting stress management and wellbeing in educators: Feasibility and efficacy of a school-based yoga and mindfulness intervention. Mindfulness, 7(1), 143-154. doi: 10.1007/s12671-0150451-2

Hue, M. T., \& Lau, N. S. (2015). Promoting well-being and preventing burnout in teacher education: A pilot study of a mindfulness-based programme for pre-service teachers in Hong Kong. Teacher Development, 19(3), 381-401. doi: 10.1080/13664530.2015.1049748

Jennings, P. A. (2015). Early childhood teachers' well-being, mindfulness, and self-compassion in relation to classroom quality and attitudes towards challenging students. Mindfulness, 6(4), 732-743. doi: 10.1007/s12671-014-0312-4

Jennings, P. A., Frank, J. L., Snowberg, K. E., Coccia, M. A., \& Greenberg, M. T. (2013). Improving classroom learning environments by cultivating awareness and resilience in education (CARE): Results of a randomized controlled trial. School Psychology Quarterly, 28(4), 374390. doi: $10.1037 /$ spq0000035

Jennings, P. A., Snowberg, K. E., Coccia, M. A., \& Greenberg, M. T. (2011). Improving classroom learning environments by cultivating awareness and resilience in education (care): Results of two pilot studies. Journal of Classroom Interaction, 46(1), 38-49. http://www.jstor.org/stable/23870550

Johnson, J. R., Emmons, H. C., Rivard, R. L., Griffin, K. H., \& Dusek, J. A. (2015). Resilience training: A pilot study of a mindfulness-based program with depressed healthcare professionals. EXPLORE: The Journal of Science and Healing, 11(6), 433-444. doi: 10.1016/j.explore.2015.08.002

Joyce, K., Pabayo, R., Critchley, J., \& Bambra, C. (2010). Flexible working conditions and their effects on employee health and wellbeing. Cochrane Database of Systematic Reviews, 2 (Art. No.: CD008009). doi: 10.1002/14651858.CD008009.pub2

Kabat-Zinn, J. (1982). An outpatient program in behavioral medicine for chronic pain patients based 
on the practice of mindfulness meditation: Theoretical considerations and preliminary results. General Hospital Psychiatry, 4(1), 33-47. doi: 10.1016/0163-8343(82)90026-3

Kabat-Zinn, J. (2003). Mindfulness-based interventions in context: Past, present, and future. Clinical Psychology: Science and Practice, 10(2), 144-156. doi: 10.1093/clipsy.bpg016

Kallapiran, K., Koo, S., Kirubakaran, R., \& Hancock, K. (2015). Review: Effectiveness of mindfulness in improving mental health symptoms of children and adolescents: A metaanalysis. Child and Adolescent Mental Health, 20(4), 182-194. doi: 10.1111/camh.12113

Klatt, M. D., Buckworth, J., \& Malarkey, W. B. (2009). Effects of low-dose mindfulness-based stress reduction (MBSR-ld) on working adults. Health Education and Behavior, 36(3), 601614. doi: $10.1177 / 1090198108317627$

Klatt, M. D., Steinberg, B., \& Duchemin, A. M. (2015). Mindfulness in motion (Mim): An onsite mindfulness based intervention (mbi) for chronically high stress work environments to increase resiliency and work engagement. Journal of Visualized Experiments, 2015(101), 111. doi: $10.3791 / 52359$

Ledesma, D., \& Kumano, H. (2009). Mindfulness-based stress reduction and cancer: A metaanalysis.Psycho-Oncology, 18(6), 571-579. doi: 10.1002/pon.1400

Lutz, A., Slagter, H. A., Dunne, J. D., \& Davidson, R. J. (2008). Attention regulation and monitoring in meditation. Trends in Cognitive Sciences, 12(4), 163-169. doi: 10.1016/j.tics.2008.01.005

Malarkey, W. B., Jarjoura, D., \& Klatt, M. (2013). Workplace based mindfulness practice and inflammation: A randomized trial. Brain, Behavior, and Immunity, 27, 145-154. doi: 10.1016/j.bbi.2012.10.009

Manotas, M., Segura, C., Eraso, M., Oggins, J., \& McGovern, K. (2014). Association of brief mindfulness training with reductions in perceived stress and distress in Colombian health care professionals. International Journal of Stress Management, 21(2), 207-225. doi: $10.1037 / \mathrm{a} 0035150$

Mirsky, A., Anthony, B., Duncan, C., Ahearn, M., \& Kellam, S. (1991). Analysis of the elements of attention: A neuropsychological approach. Neuropsychology Review, 2(2), 109-145. doi: 10.1007/BF01109051

Moher, D., Liberati, A., Tetzlaff, J., \& Altman, D. G. (2009). Preferred Reporting Items for Systematic Reviews and Meta-Analyses: The PRISMA Statement. PLoS Medicine, 6(7), e1000097. doi: 10.1371/journal.pmed.1000097

Office for National Statistics (2014). Full Report: Sickness Absence in the Labour Market. London: Office for National Statistics.

Pollard, E. L., \& Davidson, L. (2001). Action research in family and early childhood: Foundations 
for child well-being. New York: UNESCO Education Sector.

Posner, M. I., \& Petersen, S. E. (1990). The attention system of the human brain. Annual Review of Neuroscience, 13(1), 25-42. doi: 10.1146/annurev.ne.13.030190.000325

Poulin, P. A., Mackenzie, C. S., Soloway, G., \& Karayolas, E. (2008). Mindfulness training as an evidenced-based approach to reducing stress and promoting well-being among human services professionals. International Journal of Health Promotion and Education, 46(2), 72- 80. doi: 10.1080/14635240.2008.10708132

Precey, M. (2015, 17 March). Teacher stress levels in England "soaring", data shows, BBC. Retrieved from http://www.bbc.co.uk/news/education-31921457

Raffone, A., \& Srinivasan, N. (2010). The exploration of meditation in the neuroscience of attention and consciousness. Cognitive Processing, 11(1), 1-7. doi: 10.1007/s10339-009-0354-z

Ramsey, A. T., \& Jones, E. E. (2015). Minding the interpersonal gap: Mindfulness-based interventions in the prevention of ostracism. Consciousness and Cognition, 31, 24-34. doi: 10.1016/j.concog.2014.10.003

Roeser, R. W., Schonert-Reichl, K. A., Jha, A., Cullen, M., Wallace, L., Wilensky, R., ... Harrison, J. (2013). Mindfulness training and reductions in teacher stress and burnout: Results from two randomized, waitlist-control field trials. Journal of Educational Psychology, 105(3), 787-804. doi: 10.1037/a0032093

Ryff, C. D. (1989). Happiness is everything, or is it? Explorations on the meaning of psychological well-being. Journal of Personality and Social Psychology, 57(6), 1069-1081. doi: 10.1037/0022-3514.57.6.1069

Schaufeli, W. B., \& Bakker, A. B. (2003). Test manual for the Utrecht Work Engagement Scale. The Netherlands: Utrecht University

Schmidt, S., Simshäuser, K., Aickin, M., Lüking, M., Schultz, C., \& Kaube, H. (2010). Mindfulnessbased stress reduction is an effective intervention for patients suffering from migraineResults from a controlled trial. European Journal of Integrative Medicine, 2(4), 196-196. doi: 10.1016/j.eujim.2010.09.052

Schussler, D. L., Jennings, P. A., Sharp, J. E., \& Frank, J. L. (2015). Improving teacher awareness and well-being through CARE: A qualitative analysis of the underlying mechanisms. Mindfulness, 7(1), 130-142. doi: 10.1007/s12671-015-0422-7

Segal, Z. V., Williams, J. M. G., \& Teasdale, J. D. (2002). Mindfulness-based cognitive therapyfor depression: A new approach to preventing relapse. New York: Guilford Press.

Shapiro, S. L., Astin, J. A., Bishop, S. R., \& Cordova, M. (2005). Mindfulness-based stress reduction for health care professionals: Results from a randomized trial. International Journal of Stress Management, 12(2), 164-176. doi: 10.1037/1072-5245.12.2.164 
Shapiro, S. L., Carlson, L. E., Astin, J. A., \& Freedman, B. (2006). Mechanisms of mindfulness. Journal of Clinical Psychology, 62(3), 373-386. doi: 10.1002/jclp.20237

Shapiro, S. L., Schwartz, G. E., \& Bonner, G. (1998). Effects of mindfulness-based stress reduction on medical and premedical students. Journal of Behavioral Medicine, 21(6), 581-599. doi: 10.1023/A:1018700829825

Taylor, C., Harrison, J., Haimovitz, K., Oberle, E., Thomson, K., Schonert-Reichl, K., \& Roeser, R.W. (2015). Examining ways that a mindfulness-based intervention reduces stress in public school teachers: A mixed-methods study. Mindfulness, 7(1), 115-129. doi: 10.1007/s12671015-0425-4

Tirri, K. (2011). Holistic school pedagogy and values: Finnish teachers' and students' perspectives. International Journal of Educational Research, 50(3), 159-165. doi: 10.1016/j.ijer.2011.07.010

National Collaborating Centre for Methods and Tools (2008). Quality Assessment Tool for Quantiative Studies (QATQS). Hamilton, ON: McMaster University.

Vago, D. R., \& Silbersweig, D. A. (2012). Self-awareness, self-regulation, and self-transcendence (S- ART): A framework for understanding the neurobiological mechanisms of mindfulness. Frontiers in Human Neuroscience, 6, 296. doi: 10.3389/fnhum.2012.00296

Van Gordon, W., Shonin, E., Zangeneh, M., \& Griffiths, M. D. (2014). Work-related mental health and job performance: Can mindfulness help? International Journal of Mental Health and Addiction, 12(2), 129-137. doi: 10.1007/s11469-014-9484-3

Walsh, R., \& Shapiro, S. L. (2006). The meeting of meditative disciplines and western psychology: A mutually enriching dialogue. American Psychologist, 61(3), 227-239. doi: 10.1037/0003066X.61.3.227

Waters, L., Barsky, A., Ridd, A., \& Allen, K. (2015). Contemplative education: A systematic, evidence-based review of the effect of meditation interventions in schools. Educational Psychology Review, 27(1), 103-134. doi: 10.1007/s10648-014-9258-2

Weare, K. (2014). Evidence for mindfulness: Impacts on the wellbeing and performance of school staff. Exeter; Southampton: Universities of Exeter and Southampton.

World Health Organization (1948). Preamble to the Constitution of the World Health Organization as adopted by the International Health Conference, New York, 19-22 June, 1946. Geneva: World Health Organization. 


\section{Supplementary table 1}

\section{QATQS scoring assessment of intervention studies}

\begin{tabular}{|c|c|c|c|c|c|c|c|}
\hline Authors & Selection bias & Design & Cofounders & blinding & Data collection & Attrition & Global \\
\hline $\begin{array}{l}\text { Baccarani et al. } \\
\text { (2013) }\end{array}$ & 3 & 2 & 3 & 3 & 2 & 1 & 3 \\
\hline Beshai et al. (2015) & 1 & 1 & 1 & 2 & 1 & 1 & 1 \\
\hline Flook et al. (2013) & 1 & 1 & 1 & 3 & 1 & 1 & 1 \\
\hline Franco et al. (2010) & 2 & 2 & 2 & 2 & 1 & 3 & 2 \\
\hline $\begin{array}{l}\text { Frank, Riebel, et al. } \\
(2015)\end{array}$ & 3 & 2 & 2 & 3 & 1 & 3 & 3 \\
\hline Gold et al. (2010) & 3 & 2 & 3 & 3 & 2 & 2 & 3 \\
\hline Harris et al. (2015) & 2 & 2 & 2 & 2 & 1 & 1 & 2 \\
\hline Hue and Lau (2015) & 3 & 3 & 3 & 3 & 1 & 3 & 3 \\
\hline $\begin{array}{l}\text { Jennings et al. } \\
\text { (2011) }\end{array}$ & 3 & 3 & 3 & 3 & 1 & 2 & 3 \\
\hline $\begin{array}{l}\text { Jennings et al. } \\
\text { (2013) }\end{array}$ & 1 & 2 & 2 & 2 & 1 & 2 & 2 \\
\hline Klatt et al. (2009) & 2 & 2 & 3 & 3 & 1 & 1 & 2 \\
\hline $\begin{array}{l}\text { Malarkey et al. } \\
\text { (2013) }\end{array}$ & 2 & 2 & 3 & 2 & 1 & 1 & 2 \\
\hline Poulin et al. (2008) & 1 & 2 & 1 & 1 & 1 & 3 & 2 \\
\hline $\begin{array}{l}\text { Ramsey and Jones } \\
\text { (2015) }\end{array}$ & 2 & 2 & 1 & 2 & 1 & 1 & 2 \\
\hline Roeser et al. (2013) & 1 & 1 & 2 & 2 & 1 & 2 & 2 \\
\hline $\begin{array}{l}\text { Schussler et al. } \\
\text { (2015) }\end{array}$ & Q & Q & Q & Q & Q & Q & Q \\
\hline Taylor et al. (2015) & 1 & 1 & 2 & 2 & 1 & 2 & 2 \\
\hline
\end{tabular}

Note. $\mathrm{Q}=$ qualitative study 